\title{
Dietary Fiber: Food for Gut Microbiota
}

\author{
Muzaffar Hasan, Ajesh Kumar V, Chirag Maheshwari and S Mangaraj* \\ ICAR-Central Institute of Agriculture Engineering, Bhopal, India \\ *Corresponding Author: S Mangaraj, ICAR-Central Institute of Agriculture Engineering, Bhopal, India.
}

Received: July 01, 2019; Published: July 31, 2019

DOI: 10.31080/ASNH.2019.03.0389

\begin{abstract}
In $21^{\text {st }}$ century life is so fast, complex and stressed that everybody wants to stay fit and healthy. In our meals, we have so many food and nutritional items which provide us good nutrition. Among all the components of the diets, dietary fibers plays a very crucial and important role to maintain the individual health and fitness. Dietary fiber abundantly found in cereals and fruits, which helps us to keep the gut microbiota healthy. Microbes present in the gut extract energy from dietary fiber and formed metabolites like short chain fatty acids (SCFAs). SCFAs play a beneficial role to human physiology and prevent constipation and obesity. One has to include the right amount of dietary fibre in his diet to ensure the proper functioning of whole digestive system. In all dietary fiber intake help to maintain gut microbiota diversity and keep the individual healthy and fit.
\end{abstract}

Keywords: Dietary Fiber; Food; Gut Microbiota

\section{Introduction}

The human gut is a habitat of hundred trillions of microbes which plays major role in maintaining a healthy life. Our diet is not only essential to maintain human growth, reproduction, and health, but it also modulates and supports the symbiotic microbial communities that colonize the digestive tract-the gut microbiota. To sustain their proper balance inclusion of dietary fiber, food for the microorganisms, is must in our diet. Many serious diseases like colon cancer are result of diet lacking dietary fiber. So it is always recommended by the doctors to add sufficient amount of dietary fiber in diet irrespective of the age. Whole grains, fruits, vegetables, dried beans and lentils are some of the rich sources of the DF. Recommended dosage of dietary fiber is $25 \mathrm{~g}$ and $38 \mathrm{~g}$ for adult women and men respectively. But in today's world diet of many people's lack the recommended amount. Estimates say that only 5 percent of Americans meet their daily fiber requirements. As per the definition "Dietary fiber is the edible parts of plants or analogous carbohydrates that are resistant to digestion and absorption in the human small intestine with complete or partial fermentation in the large intestine". (AACC, 2001) Dietary fiber includes polysac- charides, oligosaccharides, lignin, and associated plant substances. Dietary fibers promote beneficial physiological effects including laxation, and/or blood cholesterol attenuation, and/or blood glucose attenuation. Dietary fiber is classified in two categories one is water soluble or well fermented DF like pectin, mucilages, gums, and another one is water insoluble or less fermented DF like cellulose, hemicellulose and lignin [1].

\section{Dietary fiber}

Dietary fiber defined as edible carbohydrates polymer that are resistant to digestion and absorption in the small intestine with complete or partial fermentation in large intestine [2]. Dietary fiber can be categorized in mainly 3 groups: non starch polysaccharides (NSPs), resistant starch (RS) and resistant oligosaccharides (ROs). Insoluble dietary fibers (like cellulose and hemicellulose) are very resistant to digestion and have fecal bulking effect. Some soluble dietary fiber digested (or fermented) by the gut microbes and produce some metabolites like short chain fatty acids (SCFAs) etc. Acetate, propionate and butyrate are some examples of SCFA. Both soluble and insoluble dietary fibers found in different sources such 
as legumes, fruits, vegetables, cereals, seeds, and nuts in different proportions (Table 1). Starchy foods such as tubers, legumes, cereals and unripe green bananas are rich in resistant starch (RS) whereas mature fruits and some vegetables are highly rich in pectin. [3]. Fruits have the higher content of soluble DF than the legumes and vegetables. Cereals have the higher content of insoluble DF than the other sources. It is studied that peoples who consumed more DFs rich meals able to prevent diabetes and obesity.

\begin{tabular}{|l|c|c|c|c|}
\hline \multirow{2}{*}{\multicolumn{2}{|c|}{$\begin{array}{c}\text { Source } \\
\text { Insoluble }\end{array}$}} & \multicolumn{3}{c|}{$\begin{array}{c}\text { Dietary Fiber (gm/100gm } \\
\text { edible portion) }\end{array}$} \\
\cline { 3 - 5 } & Soluble & Total & \\
\hline \multirow{4}{*}{ Grains } & Barley & - & - & 17.3 \\
\cline { 2 - 5 } & Wheat germ & 12.9 & 1.1 & 14.0 \\
\cline { 2 - 5 } & Corn & - & - & 13.4 \\
\hline \multirow{4}{*}{$\begin{array}{l}\text { Legumes } \\
\text { and pulses }\end{array}$} & White beans, raw & 13.4 & 4.3 & 17.7 \\
\cline { 2 - 5 } & Soybeans & - & - & 15.0 \\
\cline { 2 - 5 } & Lentils, raw & 10.3 & 1.1 & 11.4 \\
\hline \multirow{3}{*}{ Vegetables } & Bitter gourd & 13.5 & 3.1 & 16.6 \\
\cline { 2 - 5 } & Beetroot & 5.4 & 2.4 & 7.8 \\
\cline { 2 - 5 } & Eggplant & 5.3 & 1.3 & 6.6 \\
\hline \multirow{3}{*}{ Fruits } & Kiwi & 2.61 & 0.80 & 3.39 \\
\cline { 2 - 5 } & Pear & 2.0 & 1.0 & 3.0 \\
\cline { 2 - 5 } & Straw berry & 1.3 & 0.9 & 2.2 \\
\hline
\end{tabular}

Table 1: Dietary fiber contents of various food sources [4].

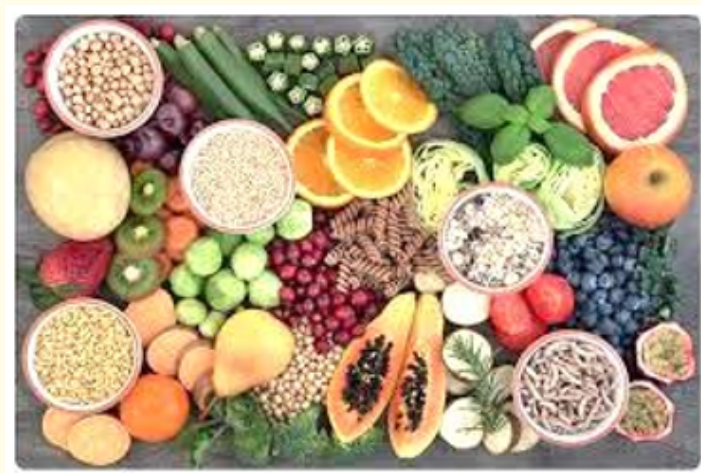

Figure a

\section{Gut microbiota}

Our digestive intestinal tract or gut harbors trillions of microbes that are essential for our physiology and development. In gut, various types of microbes are found which interact to each other and to the host. The relationship between microbes and human can be considered as mutualistic symbiosis mean healthy balance of the microbes in the host gut.

\section{Impacts of Dietary fibers on gut microbiota}

A diet has different constituents which affect the microbes in the gut. But intake of dietary fiber rich diets alter the niche of gut microbes by providing those substrates for microbial growth and help in expand the population. Different types of microbes able to produce various kinds of enzymes which make them enable to work on large number of substrates based on availability. Fermicutes and Actinobacteria are mainly response to dietary fiber. Ruminococcus bromii bacteria respond to resistant starch (RS) and initiate the breakdown of it. Dietary fiber breakdown or conversions in final metabolites take place in sequential process. For instance, Ruminococcus bromii is keystone species because it identifies and initiates the degradation of resistant starch (RS) into butyrate (SCFA) but individually unable to produce butyrate. Similarly other bacteria also work as keystone species for other type of dietary fibers but yet not to be identified [5]. Dietary fibers are notable energy source for gut microbiota by providing SCFAs especially butyrate and help in cell proliferation. Dietary fibers and SCFAs are assisting to protect the intestinal wall by secreting mucus in the gut.

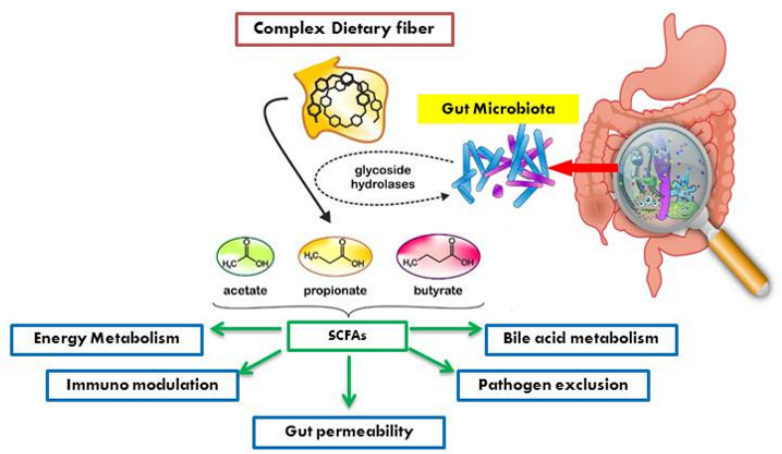

Figure 1: Role of gut microbiota in human physiology through metabolites production. 
Low dietary fiber intake loss the microbial diversity in the gut. Reduction in intake of dietary fibers instigate the microbes to switch to their less favorable substrate like protein and consequently produce detrimental metabolites for instance nitrogen containing compounds, phenolic compounds including p-cresol. These detrimental metabolites have cytotoxic and pro-inflammatory tendency and bestow the advancement of chronic disease particularly CRC (colorectal cancer).

\section{Conclusion}

Eat more fiber you have probably heard it before and in this article we tried to explain briefly why is it so? Dietary fibers rich meals have beneficial effect on gut microbiota and ultimately improve the host physiology and development. In $21^{\text {st }}$ century nobody wants to waste his precious time in toilet so high DFs rich meals consumption have the best ability to prevent or relieve constipation and subsequently prevent obesity, diabetes and other chronic intestinal diseases.

\section{Bibliography}

1. Anita FP and Abraham P. "Clinical dietetics and nutrition". Delhi Oxford University Press, Calcutta (1997): 73-77.

2. Yang J., et al. "Novel development and characterization of dietary fiber from yellow soybean hulls". Food chemistry 161 (2014): 367-375.

3. Deehan EC., et al. "Modulation of the Gastrointestinal Microbiome with Nondigestible Fermentable Carbohydrates to Improve Human Health". Microbiology spectrum 5 (2017).

4. Dhingra D., et al. "Dietary fiber in foods: a review". Journal of food science and technology 49 (2012): 255-266.

5. Makki K., et al. "The impact of dietary fiber on gut microbiota in host health and disease". Cell Host and Microbe 23 (2018): 705-715.

Volume 3 Issue 8 August 2019

(C) All rights are reserved by Muzaffar Hasan., et al. 